\title{
Inhaltsverzeichnis Jahrgang 1955
}

\section{Aufsätze}

Benveniste, E.: Sur un dictionnaire étymologique du Sanskrit .

Hermann, A.: Zur ägyptischen Literatur. . . . .

- Zur ägyptischen Stilistik

Junker, Heh.: Asien und Rom

Kammenhuber, A.: Zu den altanatolischen Sprachen: Luvisch und Palāisch .

Kees, H.: Ausgrabungen in Giza . . . . . . . .

Malinine, M.: Une Chrestomatie des Textes Démotiques Anciens.

Paret, R.: Orientalistische Bibliographie . . . .

Potratz, H.: Das Kunstwerk und seine Interpreta-

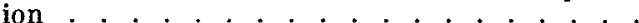

\section{Besprechungen}

Abd el-Mohsen Bakir: Slavery in Pharaonic Egypt. (W. Helck) .

Abd el-Moneim Abu-Bakr: Excavations at Giza 1949/50 (H. Kees).

Ali, S.: Anatolische Geschichten, übers. v. H. Melzig (I. Nickel) .

Alt, A.: Kleine Schriften zur Geschichte des Volkes Israel. II. (J. Lind bl om).

- Der Stadtstaat Samaria (J. Lind blo m)

Altheim, F.: Awestische Textgeschichte (H. H u mbach)

- u. R. Stiehl: Asien und Rom (Hch. Junker).

Archives Royales de Mari I-IV, s. Dossin, Jean und Kupper.

'Ārif Tãmir: Arba' rasā'il ismāî̀īya. Four Ismailite Epistles hrsg. (R. Strothmann)

Arkell, A. J.: Shaheinab (E. Ot to)

Bairel, K.: Vögel und Blumen (R. H e mpel)

Barrois, A.-G.: Manuel d'Archéologie Biblique. I. u. II (K. Galling) .

Bell, H. I.: Cults and Creeds in Graeco-Roman Egypt (K. Parlasca)

Benzing, J.: Einführung in das Studium der altaischen Philologie und der Turkologie (J.Németh)

Bloch, J.: On the Apocalyptic in Judaism (O. Ei $B^{\circ}$ feldt)

Böhl, F. M. Th. De Liagre: Het Gilgamesj-Epos. 2. Aufl. (W. v. Soden).

Böhlig, A.: Die griechischen Lehnwörter im sahidischen und bohairischen Neuen Testament mit Register und Vergleichstabellen (M. Gramer).

Bowen, R. jr.: The Early Arabian Necropolis of Ain Jawan (E. Kühnel)

Boxer, C. R.: South China in the Sixteenth Century (M. H. van der Valk) . . . . . .

Burckhardt, T.: Vom Sufitum $(\dot{A}$. Schimmel)

Burian, 0.: The Report of Lello (H. W. Duda) .

Byng, E. J.: Die Welt der Araber (R. Paret).

Caskel, W.: Lihyan und Lihyanisch (A. Dietric h).

Cassuto, U.: The Goddess Anath (O. E iBfeldt).

Cığ, M., u. Kizilyay, H.: Istanbul Arkeoloji Müzelerinde bulunan Boğazköy tabletleriIII (H.Otten)

Conze, E.: Buddhist Texts through the Ages. (J. Nobel).

Cottrell, L.: Das Geheimnis der Königsgräber (G. Roeder)

Davies, N. de Garis: Seven Private Tombs at Kurnah, ed. by A. H. Gardiner (H. W. Müller) . .

Delaporte, L., s. Huart, C.

Delougaz, P.: Pottery from the Diyala Region (A. Moortgat)
Spalte Dossin, G.: Correspondance de Samši-Addu et de ses Spalte fils transcr. et trad. (Archives Royales de Mari I und IV) (J. Denner)

485 Driver, G. R.: Semitic Writing from Pictograph to Alphabet. Rev. Ed. (O. EiBfeldt). . . . 237

97 Dussaud, R.: Prélydiens, Hittites et Achéens (A. Heubeck)

Duyvendak, J. J. L.: Tao Tö King, le livre de la voie et de la vertu (E. Erkes). . . . .

Eheling, E.: Neubabylonische Briefe Glossar zu den neubabylonischen Briefen (F. Schmidt ke)

293 - Die akkadische Gebetsserie "Handerhebung: neu hrsg. (J. Bottéro). . . . . . . . .

341 Edgerton, F.: Buddhist Hybrid Sanskrit Reader (F. Weller).

Eilers, W.: Der alte Name des persischen Neujahrsfestes (W. Hinz).

EiBfeldt, 0.: Der Gott Karmel (J. Lind blo $\mathrm{m}$ ).

- Die ältesten Traditionen Israels (W. Baumgartner) . T:

448 D'Elia, P. M., s. Ricci, M.

Elliger, K.: Studien zum Habakuk-Kommentar yom Toten Meer. - Der Hebräische Text des Habakuk-Kommentars vom Toten Meer in Umschrift (R. Meyer) .

Erichsen, W.: Auswahl frühdemotischer Texte. I: Texte; II : Umschrift u. Glossar; III: Schriftliste (M. Malinine)

Erkes, E.: Das Problem der Sklaverei in China (A. F. P. Hulsewé) .

5 Esnoul, MHe: Maitry Upanisad publ. et trad. (F. Weller).

Fakhry, A.: The Inscriptions of the Amethyst Quarries at Wadi EI Hudi (H.-W. Helck). . .

Farés, B.: Essai sur l'esprit de la décoration islamique (E. K ühnel)

79 Farrukh, 0. A., s. Sarton, G.

Field, H.: Bibliography on Southwestern Asia (A. Schall). . S

Figulla, H. H., u. Martin, W. J.: 'Ur Excavations Texts. V. (F. R. Kraus). . .

Fisehel, W. J.: Ibn Khaldūn and Josippon (R. Hartmann)

146 Forrer, L., s. Spuler, B.

Fox, P.: Tutankhamun's Treasure (W. Wolf). . . 22

45 Franke, H.: Orientalistik. I.: Sinologie (P. De miéville)

513 Franke, 0.: Geschichte des chinesischen Reiches. IV. und V. (J. Prủšek)

Frauwallner, E.: On the Date of the Buddhist Master of the Law Vasubandhu (Fr. Weller) ...

Friedrich, J.: Entzifferung verschollener Schriften und Sprachen (H. Jensen)

- Hethitisches Wörterbuch. 4. Lfg- (A. Kammenhuber).

Fürholzer, E.: China, Land und Volk (G. Köhler) .

Gabain, A. v.: Inhalt und magische Bedeutung der alttürkischen Inschriften (J. Németh) .

Gabelentz, G. von der: Chinesische Grammatik. 2. Aufl. (J. L. M. Mullie).

Gardiner, A. H., s. Davies, N. de Garis.

Glasenapp, H. v.: Das Spiel des Unendlichen (W. Kirfel).

547 Glueck, N.: Explorations in Eastern Palestine. IV,

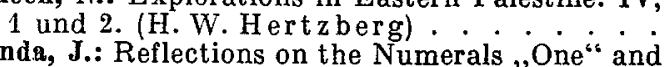
Gonda, J.: Reflections on the Numerals "One" and "Two" in Ancient Indo-European Languages Gordon, C. H.: Ugaritic Literature (A. J epsen) . 12 Grapow, H.: Anatomie und Physiologie (B.Gruber) 380 303 Hacker, P.: Vivarta (C. Regame y). . . . . 153 
Haenisch, E.: Fürst und Volk, Soldat und Beamter Spalte in Staatsnot (M. H. van der Valk). . . . . . 72 Häsler, M., s. Philips, C. H.

Handbuch der Orientalistik Bd. I: Ägyptologie II. (A. Hermann) . . . . . . . . . . . . . Harding, G. L.: Four Tomb Groups from Jordan (O. Ei Bfeldt).

Hebrew Union College Annual XXiv (O. Ei i fel $\dot{d} t)$

Hermann, A.: Die Welt der Fellachen (A. Dietrich)

Hintze, F.: Untersuchungen zu Stil und Sprache neuägyptischer Erzählungen (A. H ermann)

Höfner, M., s. Wissmann, H. von

Hölscher, U.: Post-Ramessid Remains (E. Brunner-Traut).

Holter, K.: Der Islam (A. Sc him mel)

- Persische Miniaturen (E. Kühnel)

Honigmann, E., u. A. Marieq: Recherches sur les Res Gestae divi Saporis (W. Hinz)

Hooke, S. H.: Babylonian and Assyrian Religion (W. v. Soden).

Huart, C., u. Delaporte, L.: L'Iran Antique (H. Junker)

Humbert, P.: Problèmes du livre d'Habacuc (H. Bardtke)

- La ,Terouas" (Gl. Westermann). . . . .

Hummel, S.: Geschichte der tibetischen Kunst E. Conze) . • . . . . . . . . . . . .

- Tibetisches Kunsthandwerk in Metall (P. H.

Pott) Ingholt, $_{\text {Parthian }}$ Sculptures from Hatra (O. EiBfeld t) . . . . . . . . . . .

Jäschke, G.: Der Islam in der neuen Türkei (R. Hartmann)

James, T. G. H.: The Mastaba of Khentika called Ikhekhi (H. Kees).

Jean, Ch.-F.: Lettres diverses transcr. et trad. (Archives Royales de Mari II) (J. Denner)

Jockel, R.: Götter und Dămonen (G. Mensching)

Joshi, P. S.: The Struggle for Equality (W.Ruben)

Junker, H.: Giza XI. (H. Brunner)

Kalelkar, K.: To a Gandhian Capitalist (H. L os c h)

Kapadia, D. D.: Glossary of Pahlavi Vandidad (Heh. Junker) .

Kapelrud, A. S.: Baal in the Ras Shamra Texts. (Rud. Meyer).

Kaufmann, Y.: The Biblical Account of the Conquest of Palestine (O. Ei B f eld $t$ ).

Kienitz, F. K.: Die politische Geschichte Ägyptens vom 7 . bis zum 4. Jahrhundert v. d. Z. (H. de Meulenaere)

Kisch, G.: Pseudo-Philo's Liber Antiquitatum Biblicarum (O. EiBfeldt). . . . . . . .

Kizllyay, H., s. Çı̆, M.

König, F. W.: Handbuch der chaldischen Inschriften. I. (J.Friedrich)

Komorzynski, E.: Altägypten (S. Sc $\dot{S}^{\circ} \dot{t}_{t} \dot{t}^{*} \cdot{ }^{*}$

Korvin-Krasinski, C. von: Die tibetische Medizinphilosophie (K. Quecke)

Koster, W. J. W.: Le Mythe de Platon, de Zarathoustra et des Chaldéens (F. Dornseiff).

Kracke, E. A.: Civil Service in Early Sung China $960-1067$ (J. Pr ůšek) .

Kramer, S. N., s. McCown, D. E

Krebsová, B.: Lu Sün, sa vie et son oeuvre (W. Franke).

Kreutel, R. F., u. Spies, o.: Leben und Ábenteuer des Dolmetschers Osman Aǵa (H. J. KiBling)

Kroker, E. J.: Der Gedanke der Macht im Shangkün-shu (W. Eichhorn)

Kupper, J. R.: Correspondance de $\dot{K}$ ibri-Dagan, Gouverneur de Terqa (Archives Royales de Mari III) (J. Denner)

Kurylowfez, J.: L'accentuation des langues indoeuropéennes (M. Leumann).

Lange, $\mathbf{K}$.: Sesostris (G. Lanczkowski) $\cdot \dot{*}^{*} \cdot \dot{*}^{*}$
40

\section{Lent}

poldt, J., u. S. Morenz: Heilige Schriften (W. Spalte Schubart). Lentz, W.: Iran 1951/52 (B. Spuler) . . . . . 150 wicki, M.: La Langue Mongole des Transcriptions Chinoises du XIVe Siècle (A. v. Gabain). . . 177

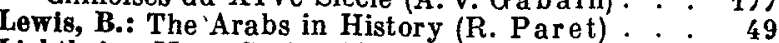
Liehtheim, M., s. Stefanski, E. †

Lindblom, J.: The Servant Songs in Deutero-Isaiah (H. W. Hertzberg) . . . . . . .

Lüders, H. †: Beobachtungen über die Sprache des buddhistischen Urkanons, hrsg. v. E. Waldschmidt (V. Pisani) .

MoCown, D. E., Steele, F. R., u. Kramer, S. N.: The New Nippur Excavations (A. Falkenstein)

Mahdi Husain: The Rehla of Ibn Battūtá (India, Maldive Islands and Ceylon) (H. M ž i k) (India,

Maricq, A., s. Honigmann, E.

Martin, W. J., s. Figulla, H. H.

Mayrhofer, M.: Handbuch des Pāli. I. u. II. (W.

- Sanskrit-Grammati

- Kurzgefaßtes etymologisches Wörterbuch des Altindischen (E. Benveniste) . . . . . . . Melzig, H., s. Ali, S.

Menon, C. A.: Catalogue of the Malayalam Manuscripts in the India Office Library (C. A. Keller)

Middleton, J.: The Central Tribes of the North Eastern Bantu (E. Dammann) . . . . . . .

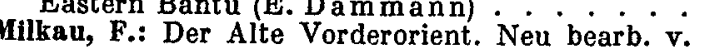
J. Schawe (H. Otten)

Moortgat, A.: Tammuz (H. Potratz). . . . . .

Tell Halaf III : Die Bildwerke (E. Douglas van Buren).

Morenz, S.: Ågypten und das Berliner Ägyptische Museum (S. Schot $t$ ). . . .

- s. Leipoldt, J.

u. J. Schubert: Der Gott auf der Blume (K. Parlasca)

Moscati, S.: Geschichte und Kultur der semitischen Völker (C. Brockelmann). . . . . Narada Thera, Le Ven.: La Doctrine Bouddhique de la Re-naissance (F. O. Schrader).

Needham, J.: Science and Civilisation in China. 1 . (W. Eichhorn) . . . . . . . . . . . . . 468

Orlinsky, H. M.: Ancient Israel (O. EiBfeldt). . . $\quad 39$

otten, H.: Keilschrifttexte aus Boghazköi. VII.

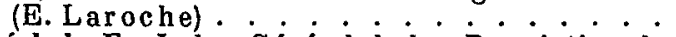

Ouéchek, E.: Index Général de la ,Description de Damas" de Sauvaire (R. Hartmann).

Parrot, A.: Déluge et Arche de Noé (O. Ei Bfeldt) - Entdeckung begrabener Welten (O. Eißfeld $t$ ). - La Tour de Babel (O. EiBfeldt).

Payne, R.: Persische Reise (W. Hinz). . $. .^{*} \cdot .^{\circ}$

Philips, C. H.: Indien. Übers. a. d. Engl. v. M. Häsler (W. Kirfel)

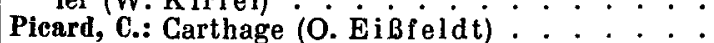

Porteous, N. W., s. Welch, A. C.

Prshewalski, N. M.: Hanhai (E. Haenisch) - . . . (eyblank, E. G.: The Background of the Rebellion

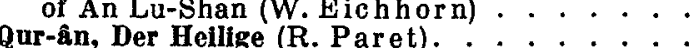
Rásonyl, L.: Sur quelques catégories de noms de personnes en Turc (A. v. Gabain)

Rathjens, C.: Sabaica I. (E. Wagner)

Renou, L.: Vocabulaire du rituel védique (j. G on $\dot{\mathrm{J}}$ a) 245

Ricci, M.: Storia dell' Introduzione del Cristianesimo in Cina, ed. P. M. D'Elia. II. u. III. (Fr. J äger)

Ricke, H.: Das Kamutef-Heiligtum in Karnak (H.-W. Helck) . . . . . . . . . . . . Robertson, E.: The old Testament Problem if.

Bardtke) Rock, J. F.: The Na-khi Näga Cult and related Cére-
. monies (J.Schubert)........... Rosenkranz, B.: Beiträge zur Erfórschung des Luvischen (A. Kammenhuber) . . . . . . . . 
Ruben, W.: Einführung in die Indienkunde (J. Fil - Spalte liozat).

Sarton, G.: The Incubation of the Western Culture in the Middle East. Transl. into Arabic by O. A. Farrukh (A. Schimme

Schawe, J., s. Milkau, F.

Sehebesta, P.: Die Negrito Asiens. I. (H. Plisch ke)

Sehmidt, P. W.: Der Ursprung der Gottesidee. X, 3 Die Religionen der Hirtenvölker. IV.: Die asiatischen Hirtenvölker (A. v. Gaba in)

Schneider, A. M. $\nmid$ : Mauern und Tore am Goldenen Horn zu Konstantinopel (F. K. Dörner) . .

Schubert, J., s. Morenz, S.

Sebestyén, I. N.: A. Sprogis' Wörterverzeichnis und grammatikalische Aufzeichnungen aus der $\mathrm{Ka}$ nin-Mundart des Jurak-Samojedischen bearb. u. hrsg. (A. J. Joki)

Seligson, M.: The Meaning of $ת$ מפ in the old Testament (Rud. Me yer)

Selms, A. v.: Marriage and Family Life in Ugaritic Literature (O. EiBfeld $t$ )

Shoukry, A.: Die Privatgrabstatue im AIten Reich (W. Wolf)

Sleg, E., u. W. Siegling: Tocharische Sprachreste Sprache B. I. und II. (W. Krause)

Siro, P.: Uber die Symmetrie des sumerischen Satze (A. Falkenstein)

Spies, 0., s. Kreutel, R. F.

Spuler, B., u. Forrer, L.: Orientalistik. III.: Der Vordere Orient in islamischer Zeit (R. Paret)

Steele, F. R., s. McCown, D. E.

Stepanski, E. $\dot{\gamma}$, u. M. Lichtheim: Coptic Ostraca from Medinet $\mathrm{Habu}$ (U. $\mathrm{Heckel}$ )

Steuernagel, C.: Hebräische Grammatik. 12. Aufl. (R. Rendt orff)

Stiehl, R., s. Altheim, $\dot{F}$.

Strothmann, R.: Morgenländische Geheimsekten in abendländischer Forschung und Die Handschrift Kiel Arab. 19 (W. B jörkman)

Tichy, H.: China ohne Mauer (G. Köhler)

Touvia Ashkenazi: A Bibliographical List of Writings 1922-1954 (O. EiBfeldt).
464

239

u. 2 (G. Roeder) . . . . . . . . Vereoutter, J.: Les objets égyptiens et égyptisants du mobilier funéraire carthaginois (K. Parlasca).

156 Vivekananda, S.: Karma-Yoga und Bhakti-Yoga (W. Ruben)

Waldschmidt, E., s. Lüiders, $\dot{H}$.

6 Wang Yi-T'ung: Official Relations between China and Japan 1368-1540 (W. Franke). . .

15 Welch, A. C.: Kings and Prophets of Israel, ed. by N. W. Porteous (H. Bardtke). . . . . . .

Weller, F.: Zwei zentralasiatische Fragmente des Buddhacarita (J. W. de J ong). . . . . . . .

204 Westendorf, W.: Der Gebrauch des Passivs in der klassischen Literatur der Ägypter (E. Ot t o).

137 Westermann, D.: Wörterbuch der Ewe-Sprache

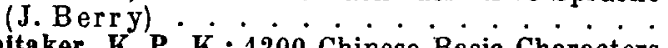
Whitaker, K. P. K.: 1200 Chinese Basic Characters (J. Buyken)

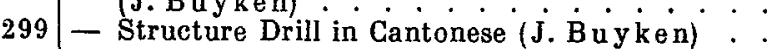

Wissmann, H. v., u. M. Höfner: Beiträge zur historischen Geographie des vorislamischen Südarabien (E. Wagner). . . . . . . . . . .

Yoshida, T.: Japanische Architektur (D. Se ckel).

Zachert, H.: Semmyō (O. Benl)

293 Zajaczkowski, A.: Słownik Arabsko-Kipczacki z okresu Państwa Mameluckiego II. (J. Benzing). . . . . . . . . . . . . .

Zbinden, E.: $\dot{D}$ ie Djinn des Islam und der altorientalische Geisterglaube (O. Spies) . . . . .

ZeitIIn, S.: The Second Book of Maccabees $(\dot{O}$. E i B feldt) . . . . . . . . . . . . . . . . . .

Zlegler, Ch.: Die Keramik von der Qal'a des Hağği

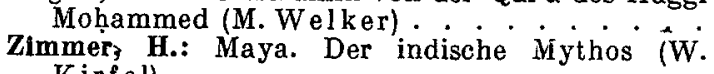
Kirfel). .................. .

[Zucker:] Festschrift für Friedrich Zucker zum 70. Geburtstage (W. Schubart). . . . . .

\section{Verzeichnis der Mitarbeiter}

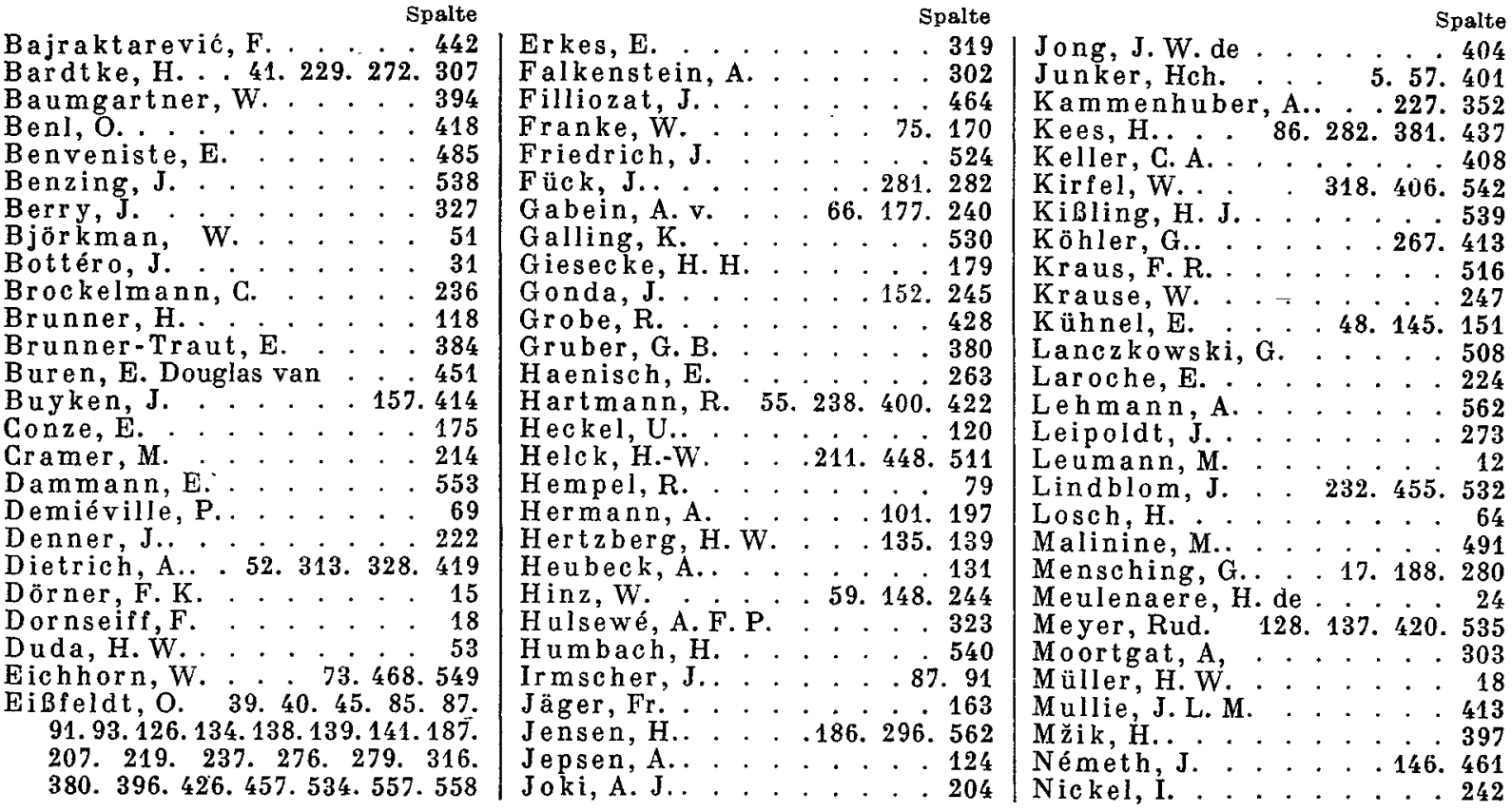




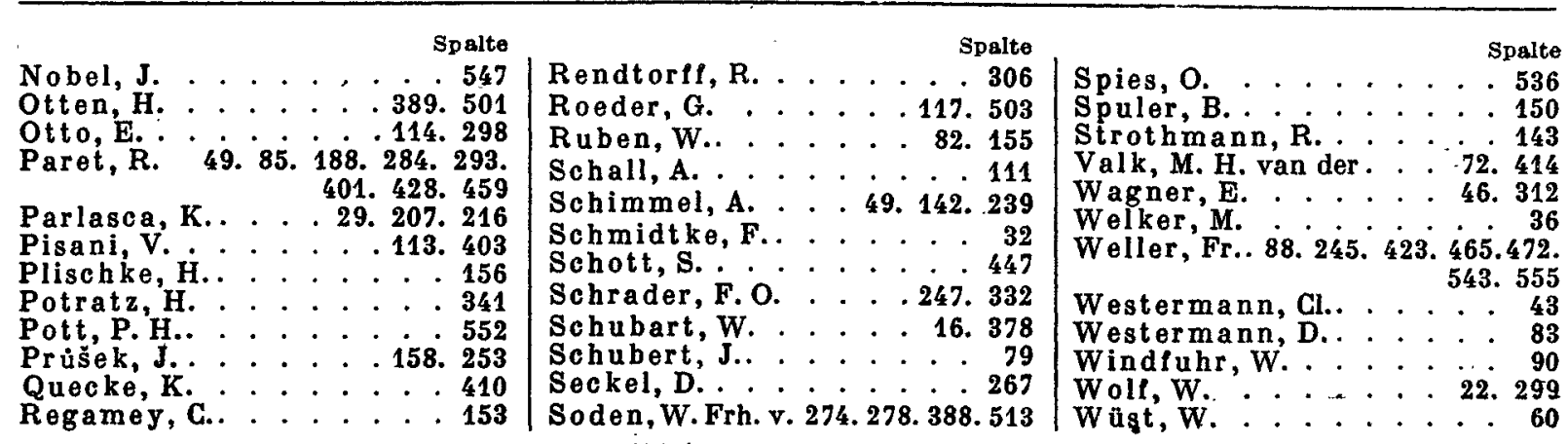

Zeltschriftenschau: Al-Mašriq 328 - Annals of the Bhandarkar Oriental Research Institute 332 - Anthropos 83 - Archiv Orientální 419 - Berytus 85 - Bulletin of the American Schools of Oriental Research $420-$ Bulletin d'Etudes Orientales 422 - Bulletin of the Faculty of Arts 85 - Bulletin de l'institut français d'archéologie orientale 86 - Bulletin of the John Rylands Library 87 - Byzantinische Zeitschrift 87 East and West 88.423 - Harvard Journal of Asiatic Studies 472. 555 - Hebrew Union College Annual 426 - The Jewish Quarterly Review 90 - Journal of the American Oriental Society 91.557 - Journal - Asiatique 179 - The Journal of Hellenic Studies 91
- Journal of Near Eastern Studies 93, 558 - Eine Heilige Kirche 562 - Language 186, 562 - Mitteilungen der Deutschen Orient-Gesellschaft 428 Nouvelle Revue Théologique 272 - Oriens Christianus 273 - Orientalia 274 - Palestine Exploration Quarterly 276 - Revue d'Assyriologie et d'Archéologie Orientale 278 - Revue Biblique 187. 279 Revue de l'Histoire des Religions 188. 280 - Studia Islamica 281 - Wiener Zeitschrift für die Kunde des Morgenlandes 282 - Zeitschrift für ägyptische Sprache und Altertumskunde 282 - Zeitschrift der Deutschen Morgenländischen Gesellschaft 188. 284. 428. 